\title{
Potential Impact of Brain and Heart Interaction on Cardiovascular System in COVID-19 Patients
}

\author{
Qianyun Guo ${ }^{1}$, Xunxun Feng ${ }^{1}$, and Yujie Zhou ${ }^{1}$ \\ ${ }^{1}$ Capital Medical University
}

July 2, 2020

\begin{abstract}
Abstract: Coronavirus disease 2019 (COVID-19), has affected many regions and countries. It is caused by the severe acute respiratory syndrome coronavirus 2 (SARS-CoV-2), which has a powerful ability to spread and is highly lethal. SARS-CoV-2 belongs to $\mathrm{CoVs}$, together with the SARS-CoV and middle east respiratory syndrome-CoV. SARS-CoV-2 damages the lungs and some other organs in humans by infecting cells via binding to the ACE2 receptor. The infection with SARS-CoV-2 results in varying degrees of clinical manifestations. Although the amount of the evidence in favor of the impact of COVID-19 on the clinical manifestations of myocardial injury and the onset of cardiovascular disease (CVD) is increasing, myocardial cell damage caused by viruses not identified in biopsies and autopsies. Therefore, it has been suggested that viruses might affect cardiac function by mechanisms other than direct infection of cardiomyocytes, and several plausible conjectures have been proposed. Based on the fact that the viruses can exert influence on multiple systems in an organism, this article describes and proposes, for the first time, the possible impact of the interaction between the brain and the heart on the cardiovascular system in COVID-19 patients.
\end{abstract}

\section{Potential Impact of Brain and Heart Interaction on Cardiovascular System in COVID-19 Patients}

Qianyun Guo*, Xunxun Feng*, Yujie Zhou

Qianyun Guo and Xunxun Feng contributed to this work equally as the first authors.

Department of Cardiology, Beijing Anzhen Hospital, Beijing Institute of Heart Lung and Blood Vessel Disease, Capital Medical University, Beijing 100029, China

Correspondence to: Dr. Yujie Zhou, Department of Cardiology, Beijing Anzhen Hospital, Capital Medical University, Beijing 100029, China (Tel: 86-10-64456489. Fax: 86-10-64442234. Email: azzyj12@163.com)

Abstract : Coronavirus disease 2019 (COVID-19), has affected many regions and countries. It is caused by the severe acute respiratory syndrome coronavirus 2 (SARS-CoV-2), which has a powerful ability to spread and is highly lethal. SARS-CoV-2 belongs to CoVs, together with the SARS-CoV and middle east respiratory syndrome-CoV. SARS-CoV-2 damages the lungs and some other organs in humans by infecting cells via binding to the ACE2 receptor. The infection with SARS-CoV-2 results in varying degrees of clinical manifestations. Although the amount of the evidence in favor of the impact of COVID-19 on the clinical manifestations of myocardial injury and the onset of cardiovascular disease (CVD) is increasing, myocardial cell damage caused by viruses not identified in biopsies and autopsies. Therefore, it has been suggested that viruses might affect cardiac function by mechanisms other than direct infection of cardiomyocytes, and several plausible conjectures have been proposed. Based on the fact that the viruses can exert influence on multiple systems in an organism, this article describes and proposes, for the first time, the possible impact of the interaction between the brain and the heart on the cardiovascular system in COVID-19 patients. 
Keywords : COVID-19; SARS-CoV-2; brain; heart; interaction

\section{Introduction}

The spread of COVID-19, a disease caused by the infection with the SARS-CoV-2, has reached pandemic proportions and dramatically impacted public health and the global economy. As of June 15, 2020, more than 7.5 million people have been infected worldwide, and more than 400,000 died. Most COVID-19 patients suffered from lung damage, but the virus has been shown to affect other systems as well. The angiotensinconverting enzyme 2 (ACE2) receptor plays a vital role in the infection of cells by the SARS-CoV-2 [1]. Although myocardial cells express ACE2 receptors, myocardial biopsies and autopsy of COVID-19 patients did not reveal significant heart damage caused by a viral infection. Despite the absence of sufficient evidence in the cardiac autopsy reports, various findings indicate that in some clinical cases, the virus may not directly attack the myocardial cells, but affect the function of the heart through alternative mechanisms. Given the paucity of reports on cardiac pathology in COVID-19, the direct impact of the virus on the heart remains unclear.

Previous studies have demonstrated a close connection between the brain and the heart, but the possibility of an interaction between these two organs in COVID-19 patients has not been raised thus far. Therefore, we would like to advance the hypothesis that that COVID-19 may affect the function of the heart through the interaction between the brain and the heart. We hope that this analysis will provide new ideas for research and suggest treatment methods that could be applied clinically.

\section{Impact of COVID-19 on the brain and the heart}

\subsection{COVID-19 and the nervous system}

COVID-19 can affect the nervous system and cause nervous system disorders. There are three major types of neurological manifestations in COVID-19 patients, involving the central nervous system, peripheral nervous system, and skeletal muscles. In comparison with mild COVID-19 cases, patients with these neurological symptoms are older and more frequently have underlying diseases, of which hypertension is the most common [2]. In a study of 417 COVID-19 patients with mild symptoms, $85.6 \%$ and $88.0 \%$ of patients had olfactory and taste dysfunction [3]. Previous reports have documented that neuroinvasiveness is a common feature of CoV. Although a specific mechanism by which SARS-CoV-2 infects neural cells was not yet known, viral RNA has been detected in the cerebrospinal fluid of COVID-19 patients. Considering the possibility of the invasion of the nervous system by SARS-CoV-2, the risk of its infection may be currently underestimated [4].

\subsection{COVID-19 in the cardiovascular system}

Because COVID-19 may affect the cardiovascular system at multiple levels, the virus infection may lead to myocardial damage and dysfunction, increasing the incidence of CVD in patients. The mechanism of COVID19-induced heart damage was unclear, but it may involve the combined effects of direct viral damage, immune damage, and cytotoxic immune cell responses in the later stages of the infection [5]. Cardiac involvement was a documented complication of COVID-19 and was associated with a higher hospital mortality rate [6]. In a study on the impact of CVD complications on death outcomes, $35.3 \%$ of 187 enrolled patients had potential CVD. Moreover, the presence of myocardial injury was significantly related to the mortality rate among COVID-19 patients, and the prognosis was better in patients without myocardial injury [7]. In a patient with acute heart injury directly related to SARS-CoV-2 localized in the myocardium, a biopsy of myocardial intima documented low-grade myocardial inflammation, but no viral particles in myocardial cells were found [8]. Therefore, the onset and progression of COVID-19 may cause myocardial damage independently of the infection of the cardiac tissue by the virus. Although the specific mechanism remained unknown, it would be still necessary to pay attention to the protection of the cardiovascular system in COVID-19 patients during the treatment of the disease.

\section{The relationship and interaction between the brain and the heart}


It is now generally accepted that there is a bidirectional interaction between the brain and the heart, i.e., the function of the brain can affect the function of the heart and vice versa [9]. The next sections summarized the possible effects of the interaction between these two organs on the cardiovascular system in COVID-19 patients, analyzed from the perspective of the autonomic nervous system and stroke.

\subsection{Autonomic nervous system}

It was well-established that brain damage can cause CVD complications, ranging from blood pressure disorders to arrhythmias. Abnormal cardiac afferent signals enhanced sustained sympathetic nerve activity and led to loss of cardiovascular protection, causing the progression of CVD, including arrhythmia-triggered sudden death [10]. Among the 416 patients hospitalized for COVID-19, almost 20\% suffered from a cardiac injury. In another study, $44 \%$ of 36 intensive care unit patients developed arrhythmia [6, 11]. Although it was currently believed that the reason for the high incidence of arrhythmia was related to myocardial injury triggered by COVID-19 [12], the possibility that the brain damage in COVID-19 patients induces arrhythmias by disturbing cardiac autonomic nervous system cannot be ignored.

\subsection{Takotsubo syndrome}

Sympathetic hyperexcitability, coronary vasospasm, and microcirculation disorders were considered the most likely factors responsible for Takotsubo syndrome (TTS). Acute cardiac dysfunction caused by TTS and subarachnoid hemorrhage might share a similar mechanism, namely the release of a large amount of catecholamines, but the understanding of the precise pathological mechanism was currently limited [13]. Although very rare, TTS might become an effective differential diagnosis of myocardial injury in COVID-19 patients. Among the reported TTS cases, one patient was confirmed to suffer from a typical SARS-CoV-2infected TTS. This finding suggested that the enormous emotional pressure and respiratory tract infection caused by the virus may be a cause of TTS [14].

\subsection{The vicious circle: stroke-heart syndrome}

Due to the extremely wide prevalence of COVID-19, the management of stroke cannot be ignored. The presence of a true relationship between COVID-19 and stroke remained to be determined. Although the number of stroke hospitalizations had declined, the evidence was available that COVID-19 infection itself may cause a stroke. It was worth noting that, without excluding the possibility of the virus directly invading the nervous system, heart injury seemed to be an important feature of this disease. The impairment of cardiac function occurs in 20-30\% of hospitalized COVID-19 patients [15]. In a pooled analysis, the cerebrovascular disease was found to be associated with a 2.5-fold increase in the severity of COVID-19 [16]. In addition, medical records from three hospitals in Wuhan indicated that $5.9 \%$ of the patients had COVID-19 infection complicated with stroke, and these patients were older, with more CVD complications, and more severe pneumonia. Stroke mechanisms in these cases might include hypercoagulable state caused by critical illness and cardiogenic embolism caused by virus-related heart damage [17]. The expression of ACE2 on vascular smooth muscle cells may also be a potential target for SARS-CoV-2 infection. The pathological analysis of COVID-19 patients documented the presence of microvascular inflammation and microthrombosis in the lungs and other affected organs [18]. Importantly, many incidences of sudden death and severe nonfatal cardiac events after the stroke may result from the interaction between cardiovascular and nervous systems [19]. Therefore, considering the possibility that COVID-19 may promote cardiac and microvascular thrombosis at various levels, the incidence of a stroke may increase with the thrombus entering the brain in COVID-19 patients. Thus, the vicious cycle in which the heart function is affected by brain damage should be considered by clinicians.

\section{COVID-19 and mental health}

COVID-19 affected not only physical but also mental health. Patients, medical staff, and the general public were under insurmountable psychological pressure. At present, it was relevant to study the means to reduce the psychological pressure associated with the COVID-19 pandemic in susceptible groups. The role of mental stress and emotional level in the development of arrhythmia and sudden cardiac death cannot be 
ignored. Of late, advances in molecular cardiology have determined the role of mutations of cardiomyocyte ion channels in arrhythmia-induced death. Negative emotional reactions may increase the risk of CVD events. Myocardial ischemia triggered by mental stress manifests with increased catecholamine concentration and increased sympathetic nerve activity, resulting in reduced coronary blood flow and ischemic changes in electrocardiograph [20]. Together, these considerations reinforce the notion that the mental state of COVID19 patients and frontline medical workers merits substantial attention. Appropriate psychological crisis intervention and psychological problem relief measures may have an essential role in the management of COVID-19. On the one hand, these approaches may improve clinical outcomes. On the other hand, they also provide first-line medical workers and the general public with psychological defense mechanisms. Therefore, active and effective intervention aiming at mental diseases and psychological conditions may mitigate CVD caused by COVID-19-related mental and psychological problems.

\section{Conclusions}

The impact of the interaction between the brain and the heart cannot be ignored. Considering that the human body is a complex integrated system, the treatment of COVID-19 patients with neurological or psychiatric symptoms requires taking into account the risk of heart damage. Although the specific mechanism by which COVID-19 affects the brain and the heart remains unknown, its combined effects on both organs may aggravate the condition of COVID-19 patients. Therefore, it is critical to monitor cardiac function in COVID19 patients developing neurological and mental disorders. It is imperative that neurologists, psychologists, and cardiologists work together on patient care. A collaborative effort can delay further development of the disease, improve the recovery rate, and reduce mortality.

\section{Acknowledgments}

In accordance with the concept of a community of shared future for mankind, confronting COVID-19 is a global battle. There is a reason to believe that the joint efforts of countries around the world will ensure effective control of the pandemic. Here, we express our deep respect and heartfelt gratitude to medical personnel working in the frontline of patient care, and to all those who zealously fight against COVID-19.

\section{Ethical Approval and Consent to participate}

Not applicable

\section{Consent for publication}

Not applicable.

\section{Availability of data and materials}

The data and materials used were all available in this perspective.

\section{Competing interests}

The authors declared that they had no competing interests.

\section{Funding}

This study was supported by grants from National Key Research and Development Program of China (2017YFC0908800), Beijing Municipal Administration of Hospitals' Ascent Plan (DFL20150601) and Mission plan (SML20180601), Beijing Municipal Health Commission "Project of Science and Technology Innovation Center" (PXM2020_026272_000005) to Y. Zhou. Q. Guo was supported by Chinese Scholarship Council (CSC) scholarship 201706210415 and China Postdoctoral Science Foundation 2019 M650032.

\section{Authors' contributions}

Qianyun Guo and Xunxun Feng wrote this perspective together and contributed to this work equally as the first authors. Yujie Zhou was corresponding author and revised this perspective. All authors read and approved the final manuscript. 


\section{References}

1 Wang Q, Zhang Y, Wu L, et al. Structural and Functional Basis of SARS-CoV-2 Entry by Using Human ACE2. Cell. 2020;181(4):894-904.e9. doi:10.1016/j.cell.2020.03.045

2 Mao L, Jin H, Wang M, et al. Neurologic Manifestations of Hospitalized Patients With Coronavirus Disease 2019 in Wuhan, China [published online ahead of print, 2020 Apr 10]. JAMA Neurol. 2020;e201127. doi:10.1001/jamaneurol.2020.1127

3 Lechien JR, Chiesa-Estomba CM, De Siati DR, et al. Olfactory and gustatory dysfunctions as a clinical presentation of mild-to-moderate forms of the coronavirus disease (COVID-19): a multicenter European study [published online ahead of print, 2020 Apr 6]. Eur Arch Otorhinolaryngol. 2020;1-11. doi:10.1007/s00405020-05965-1

4 Moriguchi T, Harii N, Goto J, et al. A first case of meningitis/encephalitis associated with SARSCoronavirus-2. Int J Infect Dis. 2020;94:55-58. doi:10.1016/j.ijid.2020.03.062

5 Zhu H, Rhee JW, Cheng P, et al. Cardiovascular Complications in Patients with COVID-19: Consequences of Viral Toxicities and Host Immune Response. Curr Cardiol Rep. 2020;22(5):32. doi:10.1007/s11886-02001292-3

6 Shi S, Qin M, Shen B, et al. Association of Cardiac Injury With Mortality in Hospitalized Patients With COVID-19 in Wuhan, China [published online ahead of print, 2020 Mar 25]. JAMA Cardiol. 2020;e200950. doi:10.1001/jamacardio.2020.0950

7 Guo T, Fan Y, Chen M, et al. Cardiovascular Implications of Fatal Outcomes of Patients With Coronavirus Disease 2019 (COVID-19) [published online ahead of print, 2020 Mar 27]. JAMA Cardiol. 2020;e201017. doi:10.1001/jamacardio.2020.1017

8 Tavazzi G, Pellegrini C, Maurelli M, et al. Myocardial localization of coronavirus in COVID-19 cardiogenic shock. Eur J Heart Fail. 2020;22(5):911-915. doi:10.1002/ejhf.1828

9 Samuels MA. The brain-heart connection. Circulation. 2007;116(1):77-84. doi:10.1161/CIRCULATIONAHA.106.678995

10 Herring N, Kalla M, Paterson DJ. The autonomic nervous system and cardiac arrhythmias: current concepts and emerging therapies. Nat Rev Cardiol. 2019;16(12):707-726. doi:10.1038/s41569-019-0221-2

11 Wang D, Hu B, Hu C, et al. Clinical Characteristics of 138 Hospitalized Patients With 2019 Novel Coronavirus-Infected Pneumonia in Wuhan, China [published online ahead of print, 2020 Feb 7]. JAMA. 2020;e201585. doi:10.1001/jama.2020.1585

12 Lazzerini PE, Boutjdir M, Capecchi PL. COVID-19, Arrhythmic Risk and Inflammation: Mind the Gap! [published online ahead of print, 2020 Apr 14]. Circulation. 2020;10.1161/CIRCULATIONAHA.120.047293. doi:10.1161/CIRCULATIONAHA.120.047293

13 Kurisu S, Kihara Y. Interaction Between Brain and Heart. Circ J. 2016;80(9):1905-1906. doi:10.1253/circj.CJ-16-0737

14 Meyer P, Degrauwe S, Van Delden C, Ghadri JR, Templin C. Typical takotsubo syndrome triggered by SARS-CoV-2 infection. Eur Heart J. 2020;41(19):1860. doi:10.1093/eurheartj/ehaa306

15 Markus HS, Brainin M. COVID-19 and stroke-A global World Stroke Organization perspective. Int J Stroke. 2020;15(4):361-364. doi:10.1177/1747493020923472

16 Aggarwal G, Lippi G, Michael Henry B. Cerebrovascular disease is associated with an increased disease severity in patients with Coronavirus Disease 2019 (COVID-19): A pooled analysis of published literature. Int J Stroke. 2020;15(4):385-389. doi:10.1177/1747493020921664 
17 AHA/ASA Stroke Council Leadership. Temporary Emergency Guidance to US Stroke Centers During the Coronavirus Disease 2019 (COVID-19) Pandemic: On Behalf of the American Heart Association/American Stroke Association Stroke Council Leadership. Stroke. 2020;51(6):1910-1912. doi:10.1161/STROKEAHA.120.030023

18 Liu PP, Blet A, Smyth D, Li H. The Science Underlying COVID-19: Implications for the Cardiovascular System [published online ahead of print, 2020 Apr 15]. Circulation. 2020;10.1161/CIRCULATIONAHA.120.047549. doi:10.1161/CIRCULATIONAHA.120.047549

19 Sörös P, Hachinski V. Cardiovascular and neurological causes of sudden death after ischaemic stroke. Lancet Neurol. 2012;11(2):179-188. doi:10.1016/S1474-4422(11)70291-5

20 Scheitz JF, Nolte CH, Doehner W, Hachinski V, Endres M. Stroke-heart syndrome: clinical presentation and underlying mechanisms. Lancet Neurol. 2018;17(12):1109-1120. doi:10.1016/S1474-4422(18)30336-3 